Open Systems \& Information Dynamics Vol. 21, No. 4 (2014) 1499001 (2 pages) DOI:10.1142/S1230161214990017

(C) World Scientific Publishing Company

\title{
Index of Vol. 21 (2014)
}

Adesso G., S. RAgy, And A. R. Lee, Continuous Variable Quantum Information: Gaussian States and Beyond

1440001

Ali M., Evolution of Genuine Multipartite Entanglement of Specific and Random States Under non-Markovian Noise

1450008

Alicki R., Quantum Thermodynamics. An Example of Two-Level Quantum Machine

1440002

Barchielli A., see Semina I., V. Semin, F. Petruccione, And A. BARCHIELLI

1440008

Benatti F., R. Floreanini, and K. Titimbo, Entanglement of Identical Particles

1440003

Bolaños-Servin J.R. And R. Carbone, Spectral Properties of Circulant Quantum Markov Semigroups

1450007

Cafaro C. And P. van Loock, A Simple Comparative Analysis of Exact and Approximate Quantum Error Correction

Carbone R., see Bolaños-Servin J. R. and R. Carbone

1450007

Chruściński D., On Kossakowski Construction of Positive Maps on Matrix Algebras

Chruściński D., On Time-Local Generators of Quantum Evolution 1440004

De Baerdemacker S., see De Vos A. and S. De Baerdemacker 1450013

De Vos A. And S. De Baerdemacker, Scaling a Unitary Matrix 1450013

Fannes M., Monotonicity of the von Neumann Entropy Expressed as a Function of Rényi Entropies

1450006

FAROOQ U. AND S. MANCINI, Information Dissipation in Random Quantum Networks

Floreanini R., see Benatti F., R. Floreanini, and K. Titimbo

1440003

Fuda T., Convergence Conditions of Mixed States and their von Neumann Entropy in Continuous Quantum Measurements

HA K.-C. And S.-H. Kye, Global Geometric Difference Between Separable and Positive Partial Transpose States

Haikka P. And S. Maniscalco, Non-Markovian Quantum Probes 
Author Index

Lee A. R., see Adesso G., S. Ragy, And A. R. Lee 1440001

Mancini S., see FAROoq U. AND S. Mancini 1450004

Maniscalco S., see HaikKa P. and S. Maniscalco 1440005

Modi K., A Pedagogical Overview of Quantum Discord 1440006

Mulalić E. H., M.S. Stanković, And R.S. Stanković, Remarks on the Pseudo-Additivity in the Axiomatization of Tsallis Entropy

1450012

NAKazato H., see TANAKA T. AND H. NAKAzATO

1440009

Ojima I., K. Okamura, And H. Saigo, Derivation of Born Rule from Algebraic and Statistical Axioms

1450005

Okamura K., see Ojima I., K. Okamura, and H. Saigo

1450005

Pascazio S., All You Ever Wanted to Know About the Quantum Zeno Effect in 70 Minutes

1440007

Pascazio S., Editorial: Introduction and Motivations

1403001

Petruccione F., see Semina I., V. Semin, F. Petruccione, And A. BARCHIELLI

1440008

Ragy S., see Adesso G., S. Ragy, and A. R. Lee 1440001

Saigo H., see Ojima I., K. Okamura, And H. SAigo 1450005

Semin V., see Semina I., V. Semin, F. Petruccione, and A. Barchielli 1440008

Semina I., V. Semin, F. Petruccione, And A. Barchielli, Stochastic Schrödinger Equations for Markovian and non-Markovian Cases

1440008

Smoliński K. A., Many Unstable Particles from an Open Quantum System's Perspective

Stanković M. S., see Mulalić E. H., M. S. Stanković, and R. S. Stanković

Stanković R. S., see Mulalić E. H., M. S. Stanković, and R. S. Stanković

TAnaka T. And H. Nakazato, Measurement of Purity, the Simplest Nonlinear Functional of the Density Matrix

Titimbo K., see Benatti F., R. Floreanini, and K. Tiтimbo 1440003

van Loock P., see CAFARO C. AND P. van LOOCK

1450002 